\title{
Neuronal variability of Resting State activity in Eating Disorders: increase and decoupling in Ventral Attention Network and relation with clinical symptoms
}

\author{
Angela V. Spalatro ${ }^{\mathrm{a}}$, Federico Amianto ${ }^{\mathrm{a}, *}$, Zirui Huang ${ }^{\mathrm{b}}$, Federico D'Agata ${ }^{\mathrm{a}}$, \\ Mauro Bergui ${ }^{c}$, Giovanni Abbate Daga ${ }^{\mathrm{a}}$, Secondo Fassino ${ }^{\mathrm{a}}$, Georg Northoff ${ }^{\mathrm{d}}$ \\ a Department of Neuroscience, Psychiatry Section, AOU City of Health and Science of Turin, University of Turin, Via Cherasco 11, 10126, Turin, Italy \\ ${ }^{\mathrm{b}}$ Department of Anesthesiology, University of Michigan Medical School, Domino Farms Lby M Ste 3100, Ann Arbor, MI, 48105-5737, USA \\ ${ }^{\mathrm{c}}$ Department of Neuroscience, Neuroradiology Section, AOU City of Health and Science of Turin, University of Turin, Via Cherasco 11, 10126, Turin, Italy \\ ' The Royal's Institute of Mental Health Research E University of Ottawa Brain and Mind Research Institute, Centre for Neural Dynamics, Faculty of Medicine, \\ University of Ottawa, 145 Carling Avenue, Rm. 6435, Ottawa, ON, K1Z 7K4 Canada
}

\section{A R T I C L E I N F O}

\section{Article history:}

Received 19 June 2018

Received in revised form 14 August 2018

Accepted 27 August 2018

Available online 29 October 2018

\section{Keywords:}

Eating Disorders

Inhibitory control

Interoceptive awareness

Neuronal variability

Ventral Attention Network

\begin{abstract}
A B S T R A C T
Background: Despite the great number of resting state functional connectivity studies on Eating Disorders (ED), no biomarkers could be detected yet. Therefore, we here focus on a different measure of resting state activity that is neuronal variability. The objective of this study was to investigate neuronal variability in the resting state of women with ED and to correlate possible differences with clinical and psychopathological indices.

Methods: 58 women respectively 25 with Anorexia Nervosa (AN), 16 with Bulimia Nervosa (BN) and 17 matched healthy controls $(\mathrm{CN})$ were enrolled for the study. All participants were tested with a battery of psychometric tests and underwent a functional Magnetic Resonance Imaging (fMRI) resting state scanning. We investigated topographical patterns of variability measured by the Standard Deviation (SD) of the Blood-Oxygen-Level-Dependent (BOLD) signal (as a measure of neuronal variability) in the restingstate and their relationship to clinical and psychopathological indices.

Results: Neuronal variability was increased in both anorectic and bulimic subjects specifically in the Ventral Attention Network (VAN) compared to healthy controls. No significant differences were found in the other networks. Significant correlations were found between neuronal variability of VAN and various clinical and psychopathological indices.

Conclusions: We here show increased neuronal variability of VAN in ED patients. As the VAN is relevant for switching between endogenous and exogenous stimuli, our results showing increased neuronal variability suggest unstable balance between body attention and attention to external world. These results offer new perspective on the neurobiological basis of ED. Clinical and therapeutic implication will be discussed.
\end{abstract}

Crown Copyright @ 2018 Published by Elsevier Masson SAS. All rights reserved.

\section{Introduction}

Eating Disorders (ED) represent the psychiatric disorder with the highest rate of mortality in adolescent and young women [1]. To date, the physiopathology of these disorders is only partially understood. Clinicians suggest a multifactorial approach, in which neurobiological factors can contribute to the vulnerability, onset and outcome of ED [2-4]. Recently a growing number of functional magnetic resonance imaging (fMRI) studies have contributed to

\footnotetext{
* Corresponding author.

E-mail address: federico.amianto@unito.it (F. Amianto).
}

clarify neuro-functional alterations in eating disorders [5]. Based on these studies, a neurobiological model of ED suggests that there could be an imbalance in information processing between ventral limbic and dorsal executive circuits [6]. These circuits are primarily implicated in inhibitory cognitive processes and reward-related behavior, which are altered in ED [7-9].

Several groups have used resting-state fMRI [10] that is thought to reflect internally oriented thinking and consciousness [11,12]. This method measures brain activity in absence of any stimulus and allows to calculate temporal correlations between different brain areas, i.e., functional connectivity $[13,14]$.

Despite the different interpretations of the Resting State studies on $\mathrm{ED}$, the results are generally overlapping in that they show 
functional alterations in various networks and/or areas. Specifically, alterations in the fronto-parietal network (a brain network involved in cognitive control functions) were found in both patients with Anorexia Nervosa (AN) and participants recovering from AN [15]. These resting-state findings may underlie the impaired cognitive control of appetitive processes as well as ruminations concerning the self and body image [16,17].

Functional connectivity describes the relationship, i.e., synchronization, between different regions and their activities in the resting state. This leaves out the neuronal features of the region itself independent of its relationship to others. For that, one could investigate the variability of neural activity in the resting state as it has been introduced recently [18-21]. This approach, called "standard deviation (SD) of blood oxygenation level-dependent (BOLD) signal" describes the fMRI fluctuations of the BOLD signal in a specific cerebral region, resulting in a more direct measure of a brain region activity [19-21]. To date, studies conducted with this approach are still very limited. Studies on psychiatric subjects show that in depression and bipolar disorders [22-27], the analysis of neuronal variability allows us to emphasize more precisely the neuro-functional alterations in terms of specific regions or networks including their relation to behavior and clinical symptoms [28,29].

The aim of this study is to explore for the first time fMRI resting state neuronal variability in ED patients including both anorectic (AN) and bulimia (BN) subjects and to correlate any differences with psychopathological and clinical symptoms. As no previous studies investigated SD in ED, our study must be considered exploratory. Based on previous findings $[7,9,12]$ we tentatively hypothesized changes in SD in specifically the attention networks like ventral attention network (VAN) in ED including their relationship to psychopathological measures.

\section{Methods and material}

\subsection{Participants}

Forty-one women who were consecutively assessed after their first contact at the Center for Eating Disorders of the University of Turin diagnosed respectively with Anorexia Nervosa (25) and Bulimia Nervosa (16) were recruited for this study between January 2012 and December 2014. A diagnosis of AN and BN was established by a psychiatrist following DSM-IV-TR criteria [30] and confirmed using DSM 5 criteria before the data elaboration. Inclusion criteria were: female sex; age of illness $<1.5$ years; righthanded; $\mathrm{BMI}<18$ and $>14$. Exclusion criteria were major medical illness, neurological disorders, use of psychotropic medications or psychotherapeutic treatments in the last 6 months, presence of other relevant psychiatric diagnosis, contraindication for MRI. Seventeen matched healthy women were enrolled for the study as control group.

\subsection{Ethics}

The study was approved by the Ethical Committee of the San Giovanni Battista Hospital, Turin. (CEI/17 0028836) according to the Helsinki Declaration as revised in Edinbourgh in 2000. All patients and controls gave their written informed consent to the study.

\subsection{Measures}

During a screening interview, an expert psychiatrist assessed every patient and control with the support of the SCID [31] and conducted a clinical evaluation to determine possible inclusion in the study. Clinical data such as length of disease, BMI, binge/ purging episodes, physical hyperactivity and use of laxative per week were also collected. According to Clinical Global Index (CGI), the psychiatrist assigned a severity index for the disease for each patient. All the participants completed a battery of self-administered psychometric tests including Temperament and Character Inventory (TCI [32]), Eating Disorder Inventory-2 (EDI-2 [33]), Toronto Alexithymia Scale (TAS-20 [34]), Symptom Checklist-90 (SCL-90 [35]), Beck Depression Inventory (BDI-II [36]) and Attachment Style Questionnaire (ASQ [37]) to evaluate psychopathological symptoms and personality features.

\section{4. fMRI data acquisition}

Functional data were collected at the Neuroscience Department AOU San Giovanni Battista, Turin, Italy on a Philips Achieva $1.5 \mathrm{~T}$ scanner (Erlangen, Holland) equipped with dual gradient system (Quasar, Philips) of $40 \mathrm{mT} / \mathrm{m}$ and a Sense high-field, high resolution eight-channel head coil optimized for functional imaging. The resting-state scan comprised 200 continuous functional volumes (repetition time $=1966 \mathrm{~ms}$, echo time $=50 \mathrm{~ms}$, flip angle $=90^{\circ}, 24$ axial slices, matrix $=128 \times 128$, slice thickness $=$ $5 \mathrm{~mm}$, acquisition voxel size $=1.8 \mathrm{~mm} \times 1.8 \mathrm{~mm} \times 6 \mathrm{~mm}$, field of view $=25 \mathrm{~cm}$ ). The total acquisition time was $6 \mathrm{~min}$ and $33 \mathrm{~s}$; the patients were scanned with their eyes closed. Foam pads were used to reduce head motion and scanner noise. All participants reported keeping their eyes closed and being awake during the scanning.

\section{5. fMRI data processing}

Preprocessing steps were implemented in Analysis of Functional NeuroImages (AFNI) software [38]. We discarded the first five volumes in each scanning using the AFNI command "3dTcat". The functional images from each scan were then slice-timing corrected (via AFNI's 3dTshift), aligned to the second volume using AFNI's “3dvolreg”, transformed into Talaraich space (Talairach J and $P$ Tournoux 1988) using and resampled to $3 \times 3 \times 3 \mathrm{~mm} 3$ using AFNI's "adwarp", and then spatially smoothed with a 6-mm full width at half maximum Gaussian blur (AFNI's "3dmerge"). Subjects with head motion larger than $\pm 3 \mathrm{~mm}$ translation or $\pm 1^{\circ}$ rotation were removed. Within our sample two subjects exceeded this threshold (they have been eliminated from the following analysis); data of all the other participants were used for the following analysis. Time series for six estimated parameters of head motion and mean time series from the white matter (WM) and cerebrospinal fluid (CSF) were regressed out from grey matter. According to previous literature, the data was then filtered with a band-pass filter preserving signals between 0.01 and $0.08 \mathrm{~Hz}$, which was thought to reflect fluctuations of spontaneous brain activity [39-41].

\subsection{Resting state analysis}

Resting-state data analysis was performed using AFNI (https:// afni.nimh.nih.gov/ afni) (38). First, the global signal variance was calculated to account for possible global changes in all subsequent analyses. Specifically, according to literature, we calculated the SD of BOLD signal changes, a measure of neuronal variability in the resting state.

Following Yang et al. [42,43] we choose to calculate the global signal using two measures, the mean of the global signal (GM) and the global signal standard deviation (GSD). This yielded no significant differences between groups and between GM and GSD. Because these measures did not reveal significant differences in each subgroups subject-level voxel-wise, SD maps $(p<.305)$ were standardized and normalized onto subject-level z-score 
maps per brain volume by subtracting the mean voxel-wise SD obtained for the entire brain (global mean of SD), then dividing by the SD across voxels [44]. That's the reason why our SD showed negative values within our sample (as reported in Fig. 1 and 2) and in all the analysis that we have done. Next, according to the literature, spherical regions of interest (ROIs) with a radius of $6 \mathrm{~mm}$ were placed in the Talairach coordinates of all cortical nodes of each network. We adopted a well-established node template from a previous study [45-47], containing 264 putative functional areas (10 $\mathrm{mm}$ diameter spheres, 32 voxels per sphere) across the whole brain. We extract 11 different networks: the Default Mode Network (DMN), the Somato Motor Network (SMN), the Salience Network (SN), the Auditory Network (AUD), the Visual Network (VS), the Dorsal Attention Network (DAN), the Ventral Attention Network (VAN), the Cerebellum Network (CB), the Frontal Parietal Temporal Network (FPTC), the Medial Regional Network (MR), the Cingular Operculum Task Control Network (COTC) and the Subcortical Network (SUB). We extracted the SD from all of the nodes and we then calculated the mean SD of all nodes within each resting-state network. Data were then filtered with two separate bands within the standard range of $0.01-0.10 \mathrm{~Hz}$ : Slow5 $(0.01-0.027 \mathrm{~Hz})$ and Slow4 $(0.027-0.073 \mathrm{~Hz})[22,23,26]$.

\subsection{Statistical analysis}

Firstly, Analysis of variance (ANOVA) and age/BDI-controlled Analysis of covariance (ANCOVA) were performed to compare all variables between $\mathrm{AN}, \mathrm{BN}$ and controls and between the two groups of subjects distinguished in patients $(\mathrm{AN}+\mathrm{BN})$ and controls using one sample t-test. A Bonferroni correction for multiple comparisons was applied accepting only results with $p<0.001$.
Only the variables that significantly differed between groups at this step were considered in the further analysis.

Secondly the SD values of the mean in each network and the SD values in each frequency band (Slow5 and Slow4) were entered into a one way ANOVA to compare group-level differences in the variability between each subgroup: AN, BN and CN. The networks' SD that were significantly different between subgroups were entered into a post hoc test to detect differences in neuronal variability between the various subgroups (i.e., $\mathrm{AN}, \mathrm{BN}$ and $\mathrm{CN}$ ). A one sample $t$-test was then performed to study differences between the two groups of subjects distinguished in patients $(\mathrm{AN}+\mathrm{BN})$ and controls. Third, a Pearson correlation analysis was performed between SD and the clinical variables (BMI and hyperactivity) and TCI, EDI-2, TAS-20, ASQ, SCL-90 subscales which differed between probands and controls.

Unless otherwise stated, all resulting t-maps were thresholded at a corrected $\mathrm{p}$-value of $<0.05$. That is, the multiple-comparison error was corrected using Monte Carlo simulation as implemented in AFNI program AlphaSim, yielding a family-wise error rate (FWER) at $\mathrm{p}<0.05$ with a minimal cluster size of 97 voxels. The smoothness used in the AlphaSim was the average smoothness across subjects. The statistical analyses were performed using Statistical Package for Social Sciences (SPSS) software (SPSS Inc., Chicago, IL, USA).

\section{Results}

\subsection{Psychopathological assessment}

We observed significant differences between anorectic subjects, bulimic subjects and healthy controls in education $[F=6.296$;

\section{Networks Distribution}

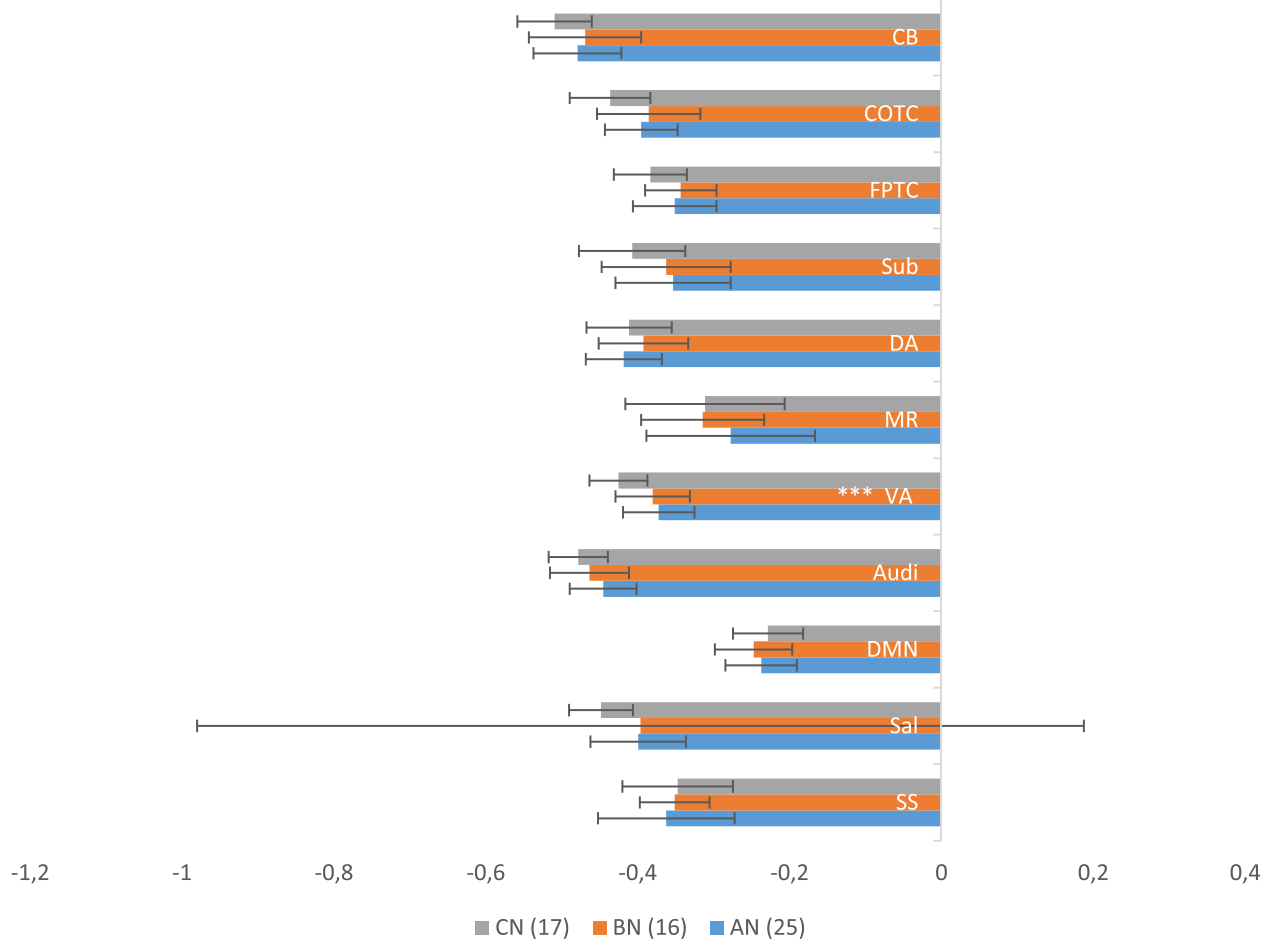

Fig. 1. Networks distribution across groups and differences between $\mathrm{AN}, \mathrm{BN}$ and $\mathrm{CN}$.

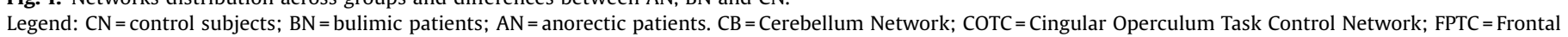

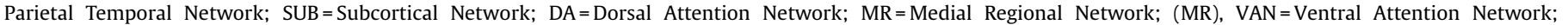
AUD = Auditory Network; DMN = Default Mode Network; SAL = Salience Network; SS = Somato Sensitive Network.

$* * * \mathrm{p}<.05$. 

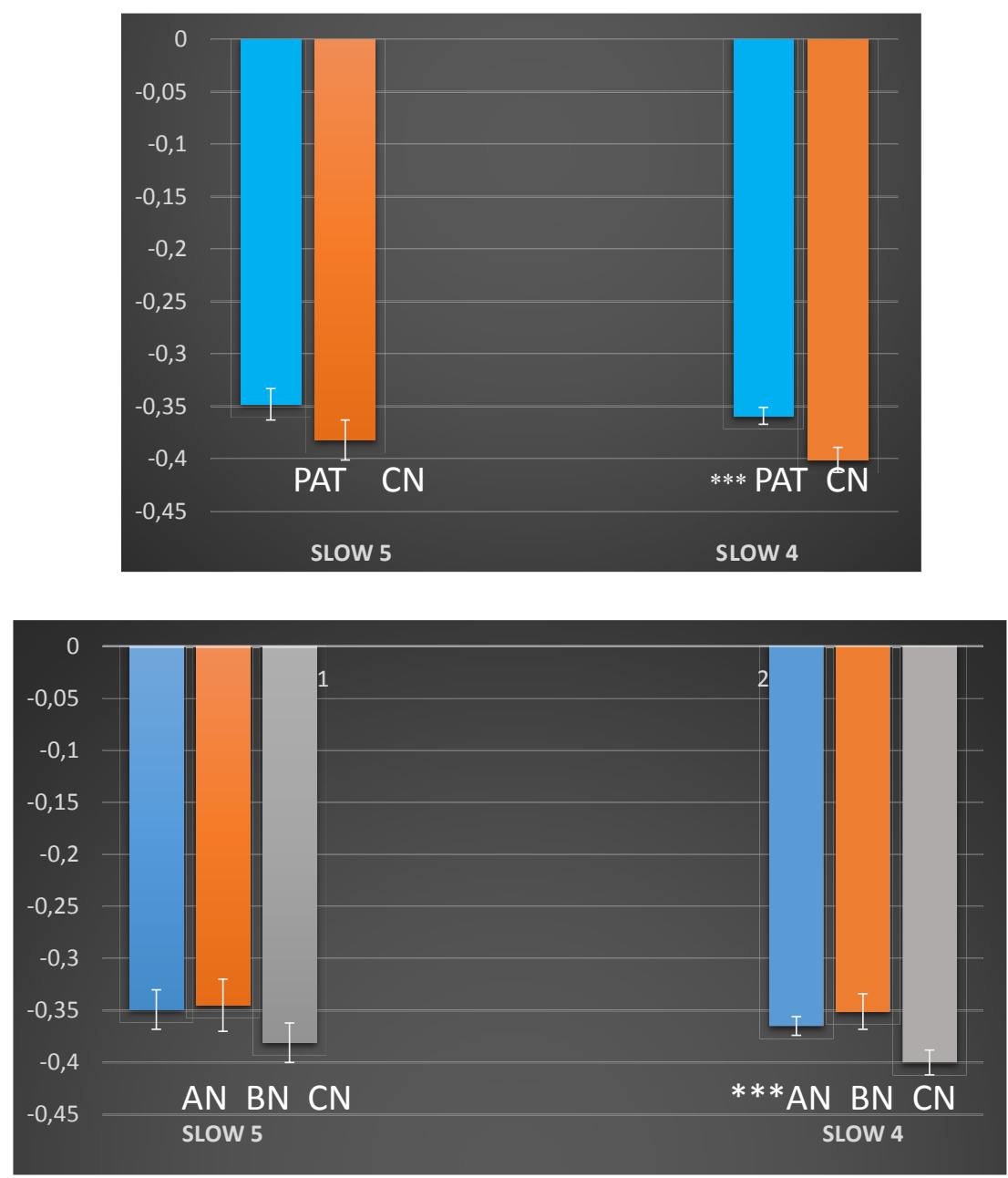

Fig. 2. SLOW 5 SLOW 4 differentiation between Patients $(A N+B N)$ and Control (2a) and between $A N, B N$ and $C N(2 b)$.

Legend: Fig. 2a. Comparison of Slow 5 and Slow 4 SD values between Patients (PAT = Anorectic patients and Bulimic Patients) and Controls (CN).

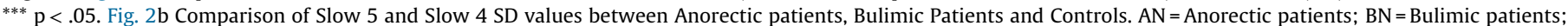
$\mathrm{CN}=$ healthy controls.

*** $\mathrm{p}<.05$

$p<0.004]$, in BMI $[F=68.856 ; p<0.000]$ and physical hyperactivity $[F=10.829 ; p<0.000]$. (Table 1 ). Some subscales of the EDI-2 (drive to thinness $[F=34.382 ; p<0.000]$, social insecurity $[F=12.073$; $p<0.000]$, interpersonal distrust $[F=10.652 ; p<0.000]$ and interoceptive awareness $[F=16.220 ; p<0.000]$ ) showed significant differences between the three groups with both anorectic and bulimic subjects scoring higher values than healthy subjects (Table 1). Moreover, both anorectic and bulimic subjects also

Table 1

Demographical, clinical and psychopathological characteristicsof the sample.

\begin{tabular}{|c|c|c|c|c|c|c|}
\hline & AN (25) & $\mathrm{BN}(16)$ & $\mathrm{CN}(17)$ & $\mathrm{F}$ & $\mathrm{p}$ & Post hoc \\
\hline Age & $20.33 \pm 4.42$ & $21.56 \pm 2.35$ & $23.27 \pm 2.19$ & 2.382 & .102 & - \\
\hline Education & $13.57 \pm 2.29$ & $14.50 \pm 2.09$ & $16.13 \pm 1.85$ & 6.296 & .004 & $\mathrm{CN}>\mathrm{AN}$ \\
\hline BMI & $15.99 \pm 0.92$ & $21.84 \pm 2.35$ & $21.42 \pm 1.85$ & 68.856 & .000 & $\mathrm{BN}, \mathrm{CN}>\mathrm{AN}$ \\
\hline Physical hyperactivity & $5.00 \pm 3.31$ & $4.86 \pm 3.46$ & $0.92 \pm 1.16$ & 10.829 & .000 & $\mathrm{AN}, \mathrm{BN}>\mathrm{CN}$ \\
\hline \multicolumn{7}{|l|}{ EDI-2 } \\
\hline Drive of thinness & $13.19 \pm 6.72$ & $16.50 \pm 6.00$ & $1.07 \pm 1.33$ & 34.382 & .000 & $\mathrm{AN}, \mathrm{BN}>\mathrm{CN}$ \\
\hline Bulimia & $3.19 \pm 4.02$ & $10.69 \pm 5.49$ & $1.20 \pm 2.14$ & 23.380 & .000 & $\mathrm{BN}>\mathrm{CN}, \mathrm{AN}$ \\
\hline Body dissatisfaction & $12.52 \pm 6.71$ & $20.62 \pm 5.33$ & $5.33 \pm 5.34$ & 23.618 & .000 & $\begin{array}{l}\mathrm{BN}>\mathrm{AN} \\
\mathrm{BN}, \mathrm{AN}>\mathrm{CN}\end{array}$ \\
\hline Impulsivity & $7.23 \pm 6.71$ & $7.31 \pm 5.39$ & $0.67 \pm 0.82$ & 8.469 & .001 & $\mathrm{AN}, \mathrm{BN}>\mathrm{CN}$ \\
\hline Social Insecurity & $6.79 \pm 4.95$ & $7.87 \pm 4.16$ & $1.27 \pm 1.83$ & 12.073 & .000 & $\mathrm{AN}, \mathrm{BN}>\mathrm{CN}$ \\
\hline Interpersonal distrust & $6.47 \pm 4.85$ & $7.93 \pm 4.52$ & $1.47 \pm 1.84$ & 10.652 & .000 & $\mathrm{AN}, \mathrm{BN}>\mathrm{CN}$ \\
\hline Interoceptive Awareness & $9.47 \pm 6.60$ & $12.18 \pm 7.02$ & $0.87 \pm 1.46$ & 16.220 & .000 & $\mathrm{AN}, \mathrm{BN}<\mathrm{CN}$ \\
\hline \multicolumn{7}{|l|}{ TAS-20 } \\
\hline Difficulty in identifying feelings & $23.95 \pm 6.13$ & $23.31 \pm 6.01$ & $10.93 \pm 4.11$ & 27.916 & .000 & $\mathrm{CN}<\mathrm{AN}, \mathrm{BN}$ \\
\hline Difficulty in describing feeling & $17.82 \pm 4.86$ & $16.93 \pm 5.64$ & $11.07 \pm 5.68$ & 7.818 & .001 & $\mathrm{CN}<\mathrm{AN}, \mathrm{BN}$ \\
\hline Externally Oriented Thinking & $17.82 \pm 4.86$ & $16.93 \pm 5.64$ & $11.07 \pm 5.68$ & 7.818 & .001 & $\mathrm{CN}<\mathrm{AN}, \mathrm{BN}$ \\
\hline Total & $59.41 \pm 9.57$ & $60.38 \pm 16.43$ & $36.73 \pm 10.41$ & 19.150 & .000 & $\mathrm{CN}<\mathrm{AN}, \mathrm{BN}$ \\
\hline
\end{tabular}

$\mathrm{AN}=$ anorectic patients; $\mathrm{BN}=$ bulimic patients; $\mathrm{CN}=$ control subjects. $\mathrm{BMI}=$ Body Mass Index; EDI-2= Eating Disorder Inventory 2; TAS=Toronto Alexithymia Scale-20. 
showed higher alexithymia scores compared with healthy subjects $[F=19.150 ; p<0.000]$ (Table 1 ). Anorectic and bulimic patients showed higher scores in many other psychopathological scales and personality dimensions (Table 2). TCI scales (persistence, cooperativeness and self-transcendence) and ASQ subscales (second nature of relationships and discomfort about intimacy) were nonsignificantly different.

\subsection{Head motion analysis}

In order to exclude the possible bias of head motion, we firstly calculated the mean of motion shift and motion rotation of the whole sample (shift: $0.06 \pm 0.03$; rotation: $0.06 \pm 0.02$ ) and then we conducted an ANOVA to detect if there are any differences between groups. No significant differences were detected between $\mathrm{AN}, \mathrm{BN}$ and healthy subjects in terms of motion shift ( $\mathrm{AN}=0.06$ $\pm 0.02 ; \mathrm{BN}=0.07 \pm 0.02 ; \mathrm{CN}=0.06 \pm 0.03 \mathrm{p}<.319)$ and motion rotation $\quad(\mathrm{AN}=06 \pm 0.02 ; \quad \mathrm{BN}=0.06 \pm 0.01 ; \quad \mathrm{CN} \quad 0.06 \pm 0.02$; $\mathrm{p}<.320)$.

\subsection{Global signal - global neuronal variability}

In a first step, following Yang et al. [42,43] we calculated the global signal using two measures, the mean of the global signal (GM) and the global signal standard deviation (GSD). This yielded no significant differences between groups (for $\mathrm{GM}$ : $\mathrm{F}=.696$; $\mathrm{p}<.503$; for GSD: $\mathrm{F}=.871 ; \mathrm{p}<.425)$ and between $\mathrm{GM}$ and GSD $(\mathrm{t}=-1.307 ; \mathrm{p}<.305)$.

\subsection{Neural networks - neuronal variability}

We then investigated neuronal variability operationalized as standard deviation of BOLD signal changes (SD) in the resting state within the 11 networks of interest. ANOVA revealed that the SD differed significantly between anorectic subjects, bulimic subjects and healthy controls in specifically the Ventral Attention Network (VAN) $[\mathrm{F}=7.311 \mathrm{p}<0.002]$ (Fig. 1). No other significant differences were found within the other networks (Table S1).
Following the literature, we also focused on Slow-5 and Slow-4 $[22,23,48-51]$. We first performed a two-sample test between patients (anorectic and bulimic subjects together) and healthy subjects for slow 5 and slow 4 (Fig. 2 a) followed by one way ANOVA between the three group separately for slow 5 and slow 4 (Fig. 2 b). This yielded significant SD differences in VAN specifically in slow 4 between patients $(\mathrm{AN}+\mathrm{BN})$ and $\mathrm{CN}$ : $[t=-2.754 ; p<.008]$ and within the three subgroups ( $\mathrm{AN}, \mathrm{BN}$ and $\mathrm{CN}$ separately): $[F=4.12 ; p<0.02]$. No significant differences were found in slow 5 . For details see also Table S2.

\subsection{Neuronal variability in networks - relationship to psychopathology}

In a final step, we correlated the neuronal data on VAN SD with psychopathological scores. Significant results showed that altered SD in VAN directly correlated with BMI $[\mathrm{p}<0.01]$ and inversely correlated with TAS total $[\mathrm{p}<0.01]$, physical hyperactivity $[\mathrm{p}<0.04]$, drive of thinness [p $<0.03$ ] and impulsivity [p $<0.03$ ] (Table 3 ). Other correlation between SD and significantly different psychopathological scores (including some EDI-2 subscales interpersonal distrust, interoceptive awareness and interpersonal distrust, TAS-20 subscale difficulty in describing feelings, some TCI subscales novelty seeking, harm avoidance and self-directedness and ASQ subscales worry about relationship and need for approval) were non-significant.

\section{Discussion}

\subsection{Main findings}

We here investigated resting state activity in patients with Eating Disorders ED with a novel measure, that is, neuronal variability. Moreover we investigated for the first time the global signal as well as neuronal variability in specific networks. This yielded the following main findings: (i) no differences in global signal between ED and healthy subjects; (ii) increased neuronal variability in specifically the Ventral Attention Network (VAN) in

Table 2

Personality and general psychopathological assessment of the sample.

\begin{tabular}{|c|c|c|c|c|c|c|}
\hline & AN (25) & $\mathrm{BN}(16)$ & $\mathrm{CN}(17)$ & $\mathrm{F}$ & $\mathrm{p}$ & Post hoc \\
\hline \multicolumn{7}{|l|}{ TCI } \\
\hline Novelty Seeking & $17.05 \pm 6.58$ & $22.63 \pm 5.19$ & $16.67 \pm 4.65$ & 5.693 & .006 & $\mathrm{BN}>\mathrm{CN}, \mathrm{AN}$ \\
\hline Harm Avoidance & $21.29 \pm 8.13$ & $23.13 \pm 5.66$ & $14.93 \pm 7.09$ & 5.578 & .006 & $\mathrm{AN}>\mathrm{CN}, \mathrm{BN}$ \\
\hline Self-directedness & $23.38 \pm 7.43$ & $20.56 \pm 8.14$ & $32.20 \pm 11.92$ & 6.875 & .002 & $\mathrm{AN}, \mathrm{BN}<\mathrm{CN}$ \\
\hline BDI-II & $16.71 \pm 9.69$ & $17.81 \pm 9.29$ & $3.00 \pm 2.10$ & 16.348 & .000 & $\mathrm{AN}, \mathrm{BN}>\mathrm{CN}$ \\
\hline \multicolumn{7}{|l|}{ SCL-90 } \\
\hline Total & $140.95 \pm 63.43$ & $159.00 \pm 49.33$ & $49.33 \pm 27.23$ & 19.583 & .000 & $\mathrm{AN}, \mathrm{BN}>\mathrm{CN}$ \\
\hline \multicolumn{7}{|l|}{ ASQ } \\
\hline Trust & $27.33 \pm 4.83$ & $25.44 \pm 4.70$ & $33.07 \pm 5.09$ & 10.356 & .000 & $\mathrm{CN}>\mathrm{AN}, \mathrm{BN}$ \\
\hline Need for approval & $35.00 \pm 5.59$ & $35.81 \pm 8.60$ & $27.47 \pm 5.11$ & 7.810 & .001 & $\mathrm{CN}<\mathrm{BN}$ \\
\hline Worry about relationships & $27.62 \pm 5.51$ & $28.38 \pm 6.68$ & $19.13 \pm 3.16$ & 14.427 & .000 & $\mathrm{CN}<\mathrm{AN}, \mathrm{BN}$ \\
\hline
\end{tabular}

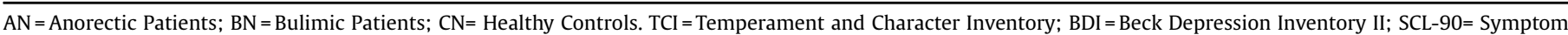
Check List-90; ASQ = Attachment Style Questionnaire.

Table 3

Correlation between VAN and psychopathology in ED patients.

\begin{tabular}{|c|c|c|c|c|c|}
\hline & $\begin{array}{l}\text { VAN } \alpha \\
\text { BMI }\end{array}$ & $\begin{array}{l}\text { VAN } \alpha \\
\text { Physical hyperactivity }\end{array}$ & $\begin{array}{l}\text { VAN } \alpha \\
\text { Drive of thinness } \\
\text { (EDI-2) }\end{array}$ & $\begin{array}{l}\text { VAN } \alpha \\
\text { Impulsivity } \\
(\text { EDI-2) }\end{array}$ & $\begin{array}{l}\text { VAN } \alpha \\
\text { Global Score } \\
\text { (TAS-20) }\end{array}$ \\
\hline Pearson & 0.389 & 0.297 & 0.322 & 0.344 & 0.372 \\
\hline $\mathrm{p}$ & 0.01 & 0.04 & 0.03 & 0.03 & 0.01 \\
\hline
\end{tabular}

ED = Eating Disorders (Anorectic patients and Bulimic patients); VAN=Ventral Attention Network; TAS-20 = Toronto Alexithymia Scale-20. 
ED; and (iii) correlation of increased neuronal variability in VAN with various psychopathological measures.

\subsection{Global signal}

We for the first time investigated the global signal (GS) in resting state of ED. Following Yang et al. [42,43], we applied two different measures of GS, that is GS mean and GS SD. Previous investigations yielded increased GS in schizophrenia on both a global level and a regional level [42,43]. In contrast, patients with bipolar disorder did not exhibit any change in the global component of GS [42]. However, a recent study showed that GS differed on the regional level in manic and depressed phases of bipolar disorder [25].

The present data investigate GS for the first time in ED. We here show no GS changes in ED on the global level. This suggests the GS to be different in ED when compared to the previous findings in schizophrenia and bipolar disorder. However, to further strengthen that claim future studies may want to directly compare ED, schizophrenia, and bipolar disorder within one and the same investigation. This perspective probably will confirm the hypothesis that GS could have a precise and clear role in the diagnostic classification of psychiatric disorders.

\subsection{Neuronal variability in ventral attention network}

Our main finding consists in increased neuronal variability in specifically the ventral attention network (VAN). The whole group of ED as well as both subgroups showed increased neuronal variability in specifically the VAN whereas no changes were observed in other networks. Moreover, we observed specific increase in slow 4, the faster frequency, whereas no such changes occurred in the slower frequency of slow 5 . This means that in ED patients the faster frequency was affected and not the slower one concerning VAN. This result differs from what has been highlighted in bipolar disorder where the slow 5 is more compromised even if this impairment concerns different networks (posterior Default Mode Network and Salience Network) [25]. This difference in the involved frequencies of the VAN may represent a further specific marker for psychiatric disorders to be explored with further studies.

Our findings are partially in contrast with a previous investigation by Collantoni et al. [52] who observed reduced functional connectivity in this network in ED. Rather than applying functional connectivity, we here measured neuronal variability which was increased in VAN and which correlated with various psychopathological measures.

The VAN is involved in sudden shifts and redirection between different stimuli [53]. In this context, VAN works as a mediator between externally- and internally-directed stimuli with their respective activities. Especially the TPJ as core region of the VAN takes on a central role in encoding body-related stimuli and their discrimination form non-bodily-related stimuli [54-56]. The impairment of neuronal variability in VAN in ED thus suggests that these patients are less able to redirect and shift their focus from body-related stimuli to non-bodily-related environmental stimuli: their attention is thus fixed and "stuck" on the own body without being able to shift their attention away from the own body. This is certainly well in line with the clinical symptom of an "increased bodily-focus" (see [57,58] for such bodily-focus in the context of depression).

Moreover, the assumption of such deficit in internal-external shifting and re-direction is further supported by our findings of correlation. Increased neuronal variability in VAN was significantly correlated with various psychopathological measures like BMI, TAS, and typical eating disorder symptoms like physical hyper intensity, drive of thinness, and impulsivity. This suggests that an impaired neuronal variability in VAN could be the basis of some clinical symptoms of patients with EDs, even if the underlying neurobiological mechanisms should be confirmed in future research.

The psychopathological symptoms in ED may thus be traced to an abnormal temporal feature in VAN neural activity, i.e., increased neuronal variability: if the neuronal activity is less variable, the likelihood of shifting and re-directing attention between internal bodily-related and external, i.e., environmental non-bodily-related stimuli is decreased so that the patients' focus remain on the internal bodily-related stimuli. That, in turn, induces the various symptoms one can observe in these patients [16,59]. Accordingly, psychopathological symptoms in ED may have temporal and spatial basis in abnormal spatiotemporal patterns of the resting state's neural activity - this amounts to what has recently been introduced as "Spatiotemporal psychopathology" $[12,60]$.

\subsection{Conclusions}

We here investigate for the first time global signal and neuronal variability in resting state in ED. While we did not find any changes in global signal in ED, our results demonstrated increased neuronal variability in a faster frequency, i.e., slow 4 in the ventral attention network in ED. Moreover, changes in resting state neuronal variability in VAN correlated with various psychopathological measures in ED. This further underlines the central relevance of VAN in ED and its altered capacity of shifting between internal body-related and external environmental non-bodily-related stimuli in ED as it can be observed in psychopathological symptoms. More generally, our findings support the recently suggested novel form of psychopathology, that is spatiotemporal psychopathology $[12,54]$ to bridge the gap between neuronal and psychopathological levels.

Two mainstreams of clinical implications may derive from our findings. The first is the possibility to support a sequential treatment of these disorders considering the impairment of the cognitive dimension of EDs as one of the possible motives of the ED psychopathology. In fact neurocognitive impairment may represent a consequence of specific neurofunctional impairment on which both relational and emotional deficits are articulated. In light of this specific therapeutic protocols such as Cognitive Remediaton Therapy may become primary-line interventions in the early stages of the ED treatment, which can be then followed by more dynamic psychotherapeutic approaches.

The second regards the possibility to act on these specific neurofunctional alterations with primarily biological instruments such as TMS, tDCS or DBS in association with psychological and nutritional therapies. In both cases the study of neurofunctional markers of EDs offers the possibility to integrate in a multidisciplinary approach both biological and psychological treatments that have been considered separately from each other from a long time.

\subsection{Limitations}

The first limitation concerns the relatively short resting state which, based on a repetition time of $2 \mathrm{~s}$, did not yield a high number of data points. Future studies may thus want to apply longer resting state with for instance $15 \mathrm{~min}$ and a lower TR (like $1 \mathrm{~s}$ ).

The second limitation concerns the relative small sample of subjects. Future studies with the same approach need to be done to confirm our preliminary results in order to generalize our hypothesis and considerations on different neurobiological aspects of ED patients. 
The third limitation concerns the use of a $1.5 \mathrm{~T}$ scanner. Future confirmatory studies should be conducted on scanners of at least $3 \mathrm{~T}$ power.

\section{Declarations of interest}

None.

\section{Contributors}

All the authors contributed to the paper. AVS contributed in sample recruitment, data collection and elaboration and in writing the paper. FA, ZH, FDA, MB, AB, GN, GAD and SF contributed in the data collection and elaboration and in the revision of the paper.

\section{Disclosures}

The lead author, AVS, affirms that the manuscript is honest, accurate, and transparent. Any discrepancies of the study have been explained within limitations.

\section{Funding source}

The present study was sponsored by the "Compagnia di San Paolo" Bank Foundation with the "Bando Neuroscienze" grant [code: 3929IT/PF 2008.2242] assigned in 2009 to Prof. Secondo Fassino and Dr. Federico Amianto and by IARPsrl generic [20BW320110719008513915033] Casale Monferrato "Resistenza ai trattamenti nei dca"

\section{Acknowledgments}

The authors thank Dr Carmen Settanta, Dr Caglio Marcella and Dr Paola Caroppo for their help in fMRI data acquisition and patients recruitment. The authors wish to acknowledge the fundings of San Paolo Bank Fundation and Cassa di Risparmio di Torino Bank Fundation given to the Professor Fassino for the support to the Pilot Centre for Eating Disorders in the years 2005-2015.

\section{Appendix A. Supplementary data}

Supplementary material related to this article can be found, in the online version, at doi:https://doi.org/10.1016/j.eurpsy.2018.08.005.

\section{References}

[1] American Psychiatric Association. Diagnostic and statistical manual of mental disorders. $5^{\text {th }}$ ed. Washington, DC: American Psychiatric Publishing; 2013.

[2] Amianto F, Northoff G, Abbate Daga G, Fassino S, Tasca GA. Is anorexia nervosa a disorder of the self? A psychological approach. Front Psychol 2016:14:7-849.

[3] Fassino S, Abbate-Daga G. Resistance to treatment in eating disorders: a critical challenge. BMC Psychiatry 2013;7:13-282.

[4] Zipfel S, Giel KE, Bulik CM, Hay P, Schmidt U. Anorexia nervosa: aetiology, assessment, and treatment. Lancet Psychiatry 2015;2:1099-111.

[5] Gaudio S, Wiemerslage L, Brooks SJ, Schiöth HB. A systematic review of resting-state functional-MRI studies in anorexia nervosa: evidence for functional connectivity impairment in cognitive control and visuospatial and body-signal integration. Neurosci Biobehav Rev 2016;71:578-89.

[6] Wagner A, Aizenstein H, Mazurkewicz L, Fudge J, Frank GK, Putnam K, et al. Altered insula response to taste stimuli in individuals recovered from restrictingtype anorexia nervosa. Neuropsychopharmacology 2008;33:513-23.

[7] D’Agata F, Caroppo P, Amianto F, Spalatro A, Caglio MM, Bergui M, et al. Brain correlates of alexithymia in eating disorders: a voxel-based morphometry study. Psychiatry Clin Neurosci 2015;69:708-16.

[8] Favaro A, Clementi M, Manara R, Bosello R, Forzan M, Bruson A, et al. CatecholO-methyltransferase genotype modifies executive functioning and prefrontal functional connectivity in women with anorexia nervosa. J Psychiatry Neurosci 2013;38:241-8.

[9] Kaye WH, Wierenga CE, Bailer UF, Simmons AN, Wagner A, Bischoff-Grethe A. Does a shared neurobiology for foods and drugs of abuse contribute to extremes of food ingestion in anorexia and bulimia nervosa? Biol Psychiatry 2013;73:836-42.

[10] Fox MD, Greicius M. Clinical applications of resting state functional connectivity. Front Syst Neurosci 2010;17:4-19.

[11] Harmelech T, Preminger S, Wertman E, Malach R. The day-after effect: long term, Hebbian-like restructuring of resting-state fMRI patterns induced by a single epoch of cortical activation. J Neurosci 2013;33:9488-97.

[12] Northoff G, Stanghellini G. How to link brain and experience? Spatiotemporal psychopathology of the lived body. Front Hum Neurosci 2016;28:10-76.

[13] Biswal BB, Mennes M, Zuo XN, Gohel S, Kelly C, Smith SM, et al. Toward discovery science of human brain function. Proc Natl Acad Sci U S A 2010;107:4734-9.

[14] Carnell S, Gibson C, Benson L, Ochner CN, Geliebter A. Neuroimaging and obesity: current knowledge and future directions. Obes Rev 2012;13:43-56.

[15] Boehm I, Geisler D, King JA, Ritschel F, Seidel M, Deza Araujo Y, et al. Increased resting state functional connectivity in the fronto-parietal and default mode network in anorexia nervosa. Front Behav Neurosci 2014;2:8-346.

[16] Abbate-Daga G, Buzzichelli S, Amianto F, Rocca G, Marzola E, McClintock SM et al. Cognitive flexibility in verbal and nonverbal domains and decision making in anorexia nervosa patients: a pilot study. BMC Psychiatry 2011:7:11-162.

[17] Canna A, Prinster A, Monteleone AM, Cantone E, Monteleone P, Volpe U, et al. Interhemispheric functional connectivity in anorexia and bulimia nervosa. Eur J Neurosci 2017;45:1129-40.

[18] Chen C, Zhou X, Chen C, Dong O, Zang Y, Qiao S, et al. The neural basis of processing anomalous information. Neuroreport 2007;18:747-51.

[19] Garrett DD, Kovacevic N, McIntosh AR, Grady CL. Blood oxygen leveldependent signal variability is more than just noise. J Neurosci 2010;30:4914-21.

[20] Garrett DD, Kovacevic N, McIntosh AR, Grady CL. The importance of being variable. J Neurosci 2011;31:4496-503.

[21] Garrett DD, Samanez-Larkin GR, MacDonald SW, Lindenberger U, McIntosh AR, Grady CL. Moment-to-moment brain signal variability: a next frontier in human brain mapping? Neurosci Biobehav Rev 2013;37:610-24.

[22] Huang Z, Davis HH, Yue Q, Wiebking C, Duncan NW, Zhang J, et al. Increase in glutamate/glutamine concentration in the medial prefrontal cortex during mental imagery: a combined functional mrs and fMRI study. Hum Brain Mapp 2015;36:3204-12.

[23] Huang Z, Zhang J, Wu J, Oin P, Wu X, Wang Z, et al. Decoupled temporal variability and signal synchronization of spontaneous brain activity in loss of consciousness: an fMRI study in anesthesia. Neuroimage 2016;124:693-703.

[24] Liu J, Corbera S, Wexler BE. Neural activation abnormalities during selfreferential processing in schizophrenia: an fMRI study. Psychiatry Res 2014;222:65

[25] Magioncalda P, Martino M, Conio B, Escelsior A, Piaggio N, et al. Functional connectivity and neuronal variability of resting state activity in bipolar disorder-reduction and decoupling in anterior cortical midline structures. Hum Brain Mapp 2015;36:666-82.

[26] Martino M, Magioncalda P, Huang Z, Conio B, Piaggio N, Duncan NW, et al. Contrasting variability patterns in the default mode and sensorimotor networks balance in bipolar depression and mania. Proc Natl Acad Sci U S A 2016;113:4824-9.

[27] Yue Y, Jia X, Hou Z, Zang Y, Yuan Y. Frequency-dependent amplitude alterations of resting-state spontaneous fluctuations in late-onset depression. Biomed Res Int 2015;505479:.

[28] Northoff G. Psychoanalysis and the brain - why did freud abandon neuroscience? Front Psychol 2012;3:71.

[29] Northoff G, Duncan NW. How do abnormalities in the brain's spontaneous activity translate into symptoms in schizophrenia? From an overview of resting state activity findings to a proposed spatiotemporal psychopathology. Prog Neurobiol 2016;146:26-45.

[30] American Psychiatric Association. Diagnostic and statistical manual of mental disorders. 4th ed., text rev. Washington, DC: American Psychiatric Publishing; 2010.

[31] First Michael B, Spitzer Robert L, Miriam Gibbon, Williams Janet, BW. Structured clinical interview for DSM-IV axis I disorders. Clinician version (SCID-CV). Washington DC: American Psychiatric Press, Inc.; 1996.

[32] Cloninger CR, Przybeck TR, Svrakic DM. The Temperament and Character Inventory (TCI): a guide to its development and use center for psychobiology of personality. Washington: University St. Louis, MO; 1996.

[33] Garner DM. Eating disorder inventory-2: professional manual. Odessa, FL: Psychological Assessment Resources; 1991.

[34] Bagby RM, Taylor GJ, Parker JD, Loiselle C. Cross-validation of the factor structure of the Toronto alexithymia scale. J Psychosom Res 1990;34:47-51.

[35] Derogatis LR. The SCL-90 manual I: scoring, administration and procedures for the SCL-90. Johns Hopkins Univ. Sch. Med. Baltim: Clin. Psychom. Unit; 1997.

[36] Beck AT, Steer RA, Brown GK, BDI-II. Beck depression inventory: manual. San Antonio, TX: Consulting Psychologists Press; 1996.

[37] Feeney JA, Noller P, Hanrahan M. Attachment in adults: clinical and developmental perspectives. Assessing adult attachment. New York: Guilford Press; 1994. p. 128-52.

[38] Cox RW. AFNI: software for analysis and visualization of functional magnetic resonance neuroimages. Comput Biomed Res 1996;29:162-73.

[39] Cordes D, Haughton VM, Arfanakis K, Carew JD, Turski PA, Moritz CH, et al. Frequencies contributing to functional connectivity in the cerebral cortex in "resting-state" data. AJNR Am J Neuroradiol 2001;22:1326-33. 
[40] Fox MD, Greicius M. Clinical applications of resting state functional connectivity. Front Syst Neurosci 2010;17:4-19.

[41] Zhang D, Raichle ME. Disease and the brain's dark energy. Nat Rev Neurol 2010;6:15-28.

[42] Yang GJ, Murray JD, Repovs G, Cole MW, Savic A, Glasser MF, et al. Altered global brain signal in schizophrenia. Proc Natl Acad Sci U S A 2014; 111:7438-43.

[43] Yang GJ, Murray JD, Wang XJ, Glahn DC, Pearlson GD, Repovs G, et al. Functional hierarchy underlies preferential connectivity disturbances in schizophrenia. Proc Natl Acad Sci U S A 2016;113:E219-28.

[44] Taylor PA, Chen G, Cox RW, Saad ZS. Open environment for multimodal interactive connectivity visualization and analysis. Brain Connect 2016;6:109-21.

[45] Laird AR, Eickhoff SB, Li K, Robin DA, Glahn DC, Fox PT. Investigating the functional heterogeneity of the default mode network using coordinate-based meta-analytic modeling. J Neurosci 2009;29:14496-505.

[46] Cole MW, Bassett DS, Power JD, Braver TS, Petersen SE. Intrinsic and taskevoked network architectures of the human brain. Neuron 2014;83:238-51.

[47] Talairach J, Tournoux P. Co-planar stereotaxic atlas of the human brain: 3 Dimensional proportional system: an approach to cerebral imaging. New York: Thieme Medical Publishers Inc.; 1988.

[48] Buzsáki G, Draguhn A. Neuronal oscillations in cortical networks. Science 2004;304:1926-9.

[49] Han Y, Wang J, Zhao Z, Min B, Lu J, Li K, et al. Frequency-dependent changes in the amplitude of low-frequency fluctuations in amnestic mild cognitive impairment: a resting-state fMRI study. Neuroimage 2011;55:287-95.
[50] Hoptman MJ, Zuo XN, Butler PD, Javitt DC, D’Angelo D, Mauro CJ, et al. Amplitude of low-frequency oscillations in schizophrenia: a resting state fMRI study. Schizophr Res 2010;117:13-20.

[51] Zuo XN, Di Martino A, Kelly C, Shehzad ZE, Gee DG, Klein DF, et al. The oscillating brain: complex and reliable. Neuroimage 2010;49:1432-45.

[52] Collantoni E, Michelon S, Tenconi E, Degortes D, Titton F, Manara R, et al. Functional connectivity correlates of response inhibition impairment in anorexia nervosa. Psychiatry Res 2016;247:9-16.

[53] Corbetta M, Shulman GL. Control of goal-directed and stimulus-driven attention in the brain. Nat Rev Neurosci 2002;3:201-15.

[54] Blanke O, Slater M, Serino A. Behavioral, neural, and computational principles of bodily self-consciousness. Neuron 2015;88:145-66.

[55] Geng JJ, Vossel S. Re-evaluating the role of TPJ in attentional control: contextual updating? Neurosci Biobehav Rev 2013;37:2608-20.

[56] Graziano MS, Kastner S. Human consciousness and its relationship to social neuroscience: a novel hypothesis. Cogn Neurosci 2011;2:98-113.

[57] Northoff G. Psychopathology and pathophysiology of the self in depression neuropsychiatric hypothesis. J Affect Disord 2007;104:1-14.

[58] Northoff G. Self and brain: what is self-related processing? Trends Cogn Sci (Regul Ed) 2011;15:186-7.

[59] Lang K, Lloyd S, Khondoker M, Simic M, Treasure J, Tchanturia K. Do children and adolescents with anorexia nervosa display an inefficient cognitive processing style? PLoS One 2015;10:e0131724.

[60] Northoff G, Huang Z. How do the brain's time and space mediate consciousness and its different dimensions? Temporo-spatial theory of consciousness (TTC). Neurosci Biobehav Rev 2017;80:630-45. 